\title{
The Importance and Applications of High Performance Size Exclusion Chromatography in Biopharmaceutical and Medical Device Industries
}

\author{
X. Michael Liu* \\ Global R\&D, Pfizer Inc. Richmond, Virginia 23220, USA
}

Received: October 07, 2015; Accepted: November 13, 2015; Published: November 18, 2015

*Corresponding author: X. Michael Liu, Global R\&D, Pfizer Inc. Richmond, Virginia 23220, USA, E-mail: x.michael.liu@pfizer.com

Size Exclusion Chromatography (SEC) or High Performance Size Exclusion Chromatography (HPSEC) is the most broadly accepted method for the determination of molecular weights and molecular weight distributions of macromolecules. It is a special type of liquid chromatography that separates molecules based on molecular sizes or hydrodynamic volumes and not according to partition or affinities toward the stationary phases. HPSEC is also called Gel Permeation Chromatography (GPC) in polymer science and gel filtration chromatography (GFC) in the biological sciences. Conventional HPSEC equipped with a concentration detector (i.e. refractive index, UV-vis, or photodiode array) acquires molecular weights and molecular weight distributions of analytes according to the elution volumes or a peak position calibration using a series of standards of known molecular weights and chemistry. Therefore, the obtained molecular weights and molecular weight distributions by conventional HPSEC are the relative ones. The advantages of conventional SEC are high robustness, excellent reproducibility, and ease of transfer between laboratories.

More recently, static /dynamic light scattering, viscometer, Fourier-Transform Infrared Spectroscopy (FT-IR), and Mass Spectrometry (MS) techniques have been used to couple with HPSEC systems. With these advanced detection technologies, the absolute molecular weights, molecular weight distributions, solution conformation, molecular structure, and chemical composition of the macromolecules can be readily obtained without using the column calibration as in conventional HPSEC. The drawbacks of implementing these new technologies include considerably increased instrumental costs, more time spent on learning the technology, greater dependence on subject matter expertise, and increased difficulty to transfer the methods between laboratories.

Traditionally the majority of Active Pharmaceutical Ingredients (APIs) are small molecules (no more than 500
Daltons). The qualitative and quantitative analyses of the APIs are often carried out using high performance liquid chromatography (HPLC) and HPLC-MSMS. However, synthetic polymers and naturally occurring biopolymers have become increasingly more important in pharmaceutical and medical industries over the past 30 years. These macromolecules can be used as coating/binding materials, active ingredients, drug delivery vehicles, polymerprotein drugs, polymer-drug conjugates, and other performanceenhancing agents. The U.S. Food and Drug Administration (FDA) and the regulatory agencies in other nations also step up to strengthen the characterization of polymer-based drugs, medical implants, and devices in the new drug/device approval guidelines. The new guidance requires that novel polymer-based drugs and devices be fully characterized to ensure high quality, safety, and efficacy of products. To conform to these new guidance and regulatory requirements, pharmaceutical and medical devices companies need to ensure that their product was manufactured according to validated/approved methods and it meets certain specifications such as molecular weight distribution, extractable and residual monomers, degree of substitution, polymer structure /composition, polymer properties, and biocompatibility.

With the advancement of polymer science and engineering, more advanced and targeted polymer-based drugs, implantable devices, and drug delivery systems will be explored and developed. The column technologies for HPSEC are similarly improving and progressing to be more versatile and more robust for challenging biopharmaceutical and medical applications. Although there are still some situations in which complementary separation-based techniques such as Field-Flow Fractionation (FFF), might be more suitable, I believe HPSEC with various advanced detection technologies will be playing more and more critical roles in the characterization and development of next generations of polymer-based drugs and medical devices. 